\title{
Binding Characteristics of Two Oxytocin Variants and Vasopressin at Oxytocin Receptors from Four Primate Species with Different Social Behavior Patterns
}

\author{
Jack H. Taylor, Nancy A. Schulte, Jeffrey A. French, and Myron L. Toews \\ Departments of Psychology (J.H.T., J.A.F.) and Biology (J.A.F.), Callitrichid Research Center, University of Nebraska at Omaha, \\ Omaha, Nebraska; and Department of Pharmacology and Experimental Neuroscience, University of Nebraska Medical Center, \\ Omaha, Nebraska (N.A.S., M.L.T.)
}

Received May 22, 2018; accepted July 27, 2018

\begin{abstract}
A clade of New World monkeys (NWMs) exhibits considerable diversity in both oxytocin (OT) ligand and oxytocin receptor (OTR) structure. Most notable is the variant $\mathrm{Pro}^{8}-\mathrm{OT}$, with proline instead of leucine at the eighth position, resulting in a rigid bend in the peptide backbone. A higher proportion of species that express $\mathrm{Pro}^{8}$-OT also engage in biparental care and social monogamy. When marmosets (genus Callithrix), a biparental and monogamous Pro ${ }^{8}$-OT NWM species, are administered the ancestral $\mathrm{Leu}^{8}-\mathrm{OT}$, there is no change in social behavior compared with saline treatment. However, when Pro ${ }^{8}$-OT is administered, marmosets' sociosexual and prosocial behaviors are altered. The studies here tested the hypothesis that OTR binding affinities and OT-induced intracellular $\mathrm{Ca}^{2+}$ potencies would favor the native OT ligand in OTRs from four primate species, each representing a unique
\end{abstract}

combination of ancestral lineage, breeding system, and native OT ligand: humans (Leu ${ }^{8}$-OT, monogamous, apes), macaques (Leu ${ }^{8}-\mathrm{OT}$, nonmonogamous, Old World monkey), marmosets (Pro ${ }^{8}$-OT, monogamous, NWM), and titi monkeys (Leu ${ }^{8}$-OT, monogamous, NWM). OTRs were expressed in immortalized Chinese hamster ovary cells and tested for intact-cell binding affinities for $\mathrm{Pro}^{8}$-OT, Leu ${ }^{8}$-OT, and arginine vasopressin (AVP), as well as intracellular $\mathrm{Ca}^{2+}$ signaling after stimulation with Pro ${ }^{8}-\mathrm{OT}$, Leu ${ }^{8}-\mathrm{OT}$, and AVP. Contrary to our hypothesis, Pro ${ }^{8}$-OT bound at modestly higher affinities and stimulated calcium signaling at modestly higher potencies compared with $\mathrm{Leu}^{8}$-OT in all four primate OTRs. Thus, differences downstream from a ligand-receptor binding event are more likely to explain the different behavioral responses to these two ligands.

\section{Introduction}

Oxytocin (OT) is a nonapeptide neurohormone that is critical for mammalian parturition, lactation, and parental behavior (Ellendorff et al., 1982; Fuchs et al., 1982; McNeilly et al., 1983; Chan et al., 1996; Lee et al., 2009). OT binds and activates its canonical G protein-coupled receptor, the oxytocin receptor (OTR). Synthetic OT is used widely in clinical settings for inducing and accelerating labor. Because of its ability to modulate a wide variety of social behaviors (Lee et al., 2009), OT is currently being evaluated for its clinical use in disorders with a social component, such as autism spectrum disorder and schizophrenia (Bakermans-Kranenburg and van Ijzendoorn, 2013; Feifel et al., 2016; DeMayo et al., 2017; Parker et al., 2017). Considerable effort has been invested in

This work was supported by the National Institutes of Health Eunice Kennedy Shriver National Institute of Child Health and Human Development [Grant R01HD089147] and the University of Nebraska at Omaha [Graduate Research and Creative Activity Award ("Functional Characteristics of Four Primate Oxytocin Receptors: Relationships to Biparental Care and Social Monogamy")].

https://doi.org/10.1124/jpet.118.250852. engineering OT analogs and formulations for potential therapeutic use and to extend understanding of OT actions, particularly central nervous system and behavioral effects (Manning et al., 2012; Busnelli et al., 2013; Muttenthaler et al., 2017). Although these efforts with novel synthetic analogs have been reasonably successful, naturally occurring variants of the OT peptide could provide an alternate route to novel agents and therapies (Gruber et al., 2012).

The nonapeptide family of hormone ligands is ancient and is present in nearly all animal lineages (Beets et al., 2013; Lockard et al., 2017). OT-like ligands generally vary at the third, fourth, or eighth amino acid position (Gruber et al., 2012), and the amino acid at the eighth position strongly affects the activity of the peptide on its target organs (Sawyer and Manning, 1973; Manning et al., 2012; Muttenthaler et al., 2017). OT and the closely related nonapeptide arginine vasopressin (AVP) differ at amino acid positions 3 and 8 and have vastly different roles in mammalian physiology, even though the affinity of OT for OTRs is only 2-fold greater than the affinity of AVP for OTRs (Manning et al., 2012). Despite having only a 2 -fold lower binding affinity for OTRs, AVP is over 30 -fold less potent than OT for generating OTR responses

ABBREVIATIONS: AVP, arginine-8-vasopressin; CHO, Chinese hamster ovary; HGH-BSA, high glucose HEPES-buffered Dulbecco's modified Eagle's medium containing $0.1 \%$ bovine serum albumin; NWM, New World monkey; OT, oxytocin; OTR, oxytocin receptor; OVTA, ornithine vasotocin analog. 
TABLE 1

Representative primate species OTRs

\begin{tabular}{|c|c|c|c|c|}
\hline Group & Human & Macaque & Marmoset & Titi Monkey \\
\hline Lineage (family) & Old World (Hominidae) & Old World (Cercopithidae) & New World (Callitrichidae) & New World (Pithecidae) \\
\hline Breeding system & Monogamous & Polygamous & Monogamous & Monogamous \\
\hline Native OT ligand & $\mathrm{Leu}^{8}-\mathrm{OT}$ & $\mathrm{Leu}^{8}-\mathrm{OT}$ & Pro $^{8}-\mathrm{OT}$ & $\mathrm{Leu}^{8}-\mathrm{OT}$ \\
\hline
\end{tabular}

(Manning et al., 2012). Variations among species in their nonapeptide receptors correlate with variations in their respective OT-like ligands, indicating ligand-receptor coevolution (Koehbach et al., 2013). The presence of OT-like peptides across diverse animal taxa suggests the universal importance of their functions, and their coevolution with their ligands suggests a tightly aligned signaling system for these functions.

Despite variation across Animalia taxa, the OT ligand is highly conserved within eutherian mammals (Wallis, 2012). Recently, a nonsynonymous nucleotide substitution in the $O X T$ gene coding for OT was discovered in four species of New World monkeys (NWMs), resulting in a proline at amino acid position 8 ( Pro $^{8}-\mathrm{OT}$ ) in place of the typical leucine ( $\mathrm{Leu}^{8}-\mathrm{OT}$ ) (Lee et al., 2011). Subsequent screening showed that the Pro $^{8}$-OT variant is present in at least 20 NWM species (Ren et al., 2015; Vargas-Pinilla et al., 2015). Additional OT variants were also identified, for a total of six different forms of OT in NWMs, with at least one species from each NWM clade exhibiting an OT variant (Ren et al., 2015; VargasPinilla et al., 2015). OTRs also vary in NWMs, particularly in the $\mathrm{N}$ terminus (Ren et al., 2015; Vargas-Pinilla et al., 2015), which is important for binding to the tail of the OT ligand (Postina et al., 1996; Gimpl and Fahrenholz, 2001), and there is strong evidence for OT-OTR coevolution (Koehbach et al., 2013; Ren et al., 2015; Vargas-Pinilla et al., 2015). Moreover, OXTR variation is associated with social monogamy among primates (Ren et al., 2015), and OT ligand variation at position 8 is associated with litter size within the family Cebidae (Vargas-Pinilla et al., 2015). Both native and non-native OT ligands modulate social behavior in NWMs expressing Pro ${ }^{8}$-OT (French et al., 2016), but the native Pro $^{8}$-OT is more effective at modulating behavior than the ancestral $\mathrm{Leu}^{8}-\mathrm{OT}$ in the marmoset, a monogamous and biparental NWM (Cavanaugh et al., 2014; Mustoe et al., 2015, 2018). Together these findings indicate that both OT ligand variation and the corresponding variations in OTRs among NWMs contribute to functional outcomes.

Based on the findings summarized above, we hypothesized that the binding affinities and signaling potencies of primate OT variants are different for different OTR variants, with each receptor variant preferring the ligand variant from the same species. The studies presented here test this hypothesis by measuring binding affinities and signaling potencies for Leu8, Pro8, and AVP at the OTRs from four primate species, each representing a unique combination of ancestral lineage, breeding system, and native OT ligand.

\section{Materials and Methods}

OTR Transfection and Cell Culture. Chinese hamster ovary (CHO; female origin) cells were purchased from American Type Culture Collection (Manassas, VA) and cultured at $37^{\circ} \mathrm{C}$ with $5 \%$ $\mathrm{CO}_{2}$ using Ham's F-12 medium supplemented with $10 \%$ fetal bovine serum and $100 \mathrm{U} / \mathrm{ml}$ penicillin and $100 \mu \mathrm{g} / \mathrm{ml}$ streptomycin. Human, marmoset, and macaque OTR plasmids (Table 1) were purchased from GenScript (Piscataway, NJ) in a pcDNA3.1+ vector. The titi monkey plasmid was generated by amplifying and ligating the coding region of the titi OXTR from genomic titi monkey DNA (flanked with BamHI and XhoI restriction sites) and ligating it into a $\mathrm{T}$ vector (pMD19). Competent Escherichia coli were transformed using this vector, plated onto Luria-Bertani/ampicillin/isopropyl $\beta$-D-1-thiogalactopyranoside/ $\mathrm{X}$-gal plates, and incubated overnight at $37^{\circ} \mathrm{C}$. White colonies were selected, then plasmid DNA was purified and sequenced. Sequenceconfirmed plasmids were then digested with BamHI and XhoI and ligated into a pcDNA3.1+ vector. CHO cells were then transfected using TurboFect (Thermo Fisher Scientific, Waltham, MA) according to the manufacturer's instructions and kept under selective pressure using $400 \mu \mathrm{g} / \mathrm{ml} \mathrm{G} 418$ antibiotic. Individual clonal lines were generated by plating batch-transfected cells at approximately 10 cells $/ \mathrm{ml}$ ( 1 cell/100 $\mu \mathrm{l}$ ) into 96 -well plates and then selecting wells for screening that originated from a single colony. Clonal lines were screened using an intact cell ${ }^{125}$ I-ornithine vasotocin analog (OVTA) binding assay and selected for similar receptor expression across species, defined as total radioligand binding. All experiments were done in a single clone per species, except for the marmoset, in which two clones were used.

Intact Cell Saturation Binding Assays. CHO cells expressing primate OTRs were plated at 150,000 cells $/ \mathrm{ml}(15,000$ cells/well per $100 \mu \mathrm{l}$ ) into 96 -well plates and grown to $80 \%-90 \%$ confluence. On the day of assay, growth medium was aspirated and cells were quickly washed once with $100 \mu \mathrm{l}$ ice-cold high glucose HEPES-buffered Dulbecco's modified Eagle's medium containing $0.1 \%$ bovine serum albumin (HGH-BSA) and then placed on ice. Then $50 \mu$ lice-cold ${ }^{125}$ I-OVTA (PerkinElmer, Waltham, MA) in doubling concentrations from about 15 to $2000 \mathrm{pM}$ was added in triplicate (technical replicates) to all wells and incubated for 3 hours on ice. At the end of the assay, an aliquot of the binding medium was collected to quantify free radioligand directly, eliminating any concerns about differential depletion of ligand due to differential receptor expression levels. Cells were then washed four times with $100 \mu \mathrm{l}$ ice-cold HGH-BSA, solubilized with $100 \mu \mathrm{l} 0.2 \mathrm{~N} \mathrm{NaOH}$, and counted on a gamma counter. Nonspecific binding was defined as ${ }^{125} \mathrm{I}$-OVTA binding occurring in the presence of excess competitor $\left(10^{-4} \mathrm{M} \mathrm{Leu}^{8}\right.$-OT). Binding affinity $\left(K_{\mathrm{d}}\right)$ for ${ }^{125}$ I-OVTA was determined using GraphPad Prism software (GraphPad Software Inc., La Jolla, CA) to fit the specific bound versus free ligand data to a single-site binding equation. These assays were done at least three times on 3 different days using fresh aliquots of ${ }^{125}$ I-OVTA and competitor, and $K_{\mathrm{d}}$ values were averaged across three biologic replicates (five biologic replicates for marmoset).

Intact Cell Competition Binding Assays. CHO cells expressing primate OTRs were plated at 150,000 cells $/ \mathrm{ml}(15,000$ cells/well per $100 \mu \mathrm{l}$ ) into 96 -well plates and grown to $80 \%-90 \%$ confluence. On the day of assay, growth medium was aspirated and cells were quickly washed once with $100 \mu \mathrm{l}$ ice-cold HGH-BSA and then placed on ice. Then $50 \mu \mathrm{l}$ of roughly $50,000 \mathrm{cpm}$ ice-cold ${ }^{125}$ I-OVTA was added in triplicate (technical replicates) to all wells in the presence or absence of $10^{-11}$ to $10^{-5} \mathrm{M}^{\mathrm{Pro}}{ }^{8}$-OT (CYIQNCPPG-NH2; Anaspec, Fremont, CA), Leu ${ }^{8}$-OT (CYIQNCPLG-NH2; Anaspec), or AVP (CYFQNCPRGNH2; Anaspec) and incubated for 3 hours on ice. At the end of the assay, an aliquot of the binding medium was collected to quantify free radioligand directly. Cells were then washed four times with $100 \mu \mathrm{l}$ ice-cold HGH-BSA, solubilized with $100 \mu \mathrm{l} 0.2 \mathrm{~N} \mathrm{NaOH}$, and counted on a gamma counter. Binding affinities $\left(\mathrm{IC}_{50}\right)$ were determined by 


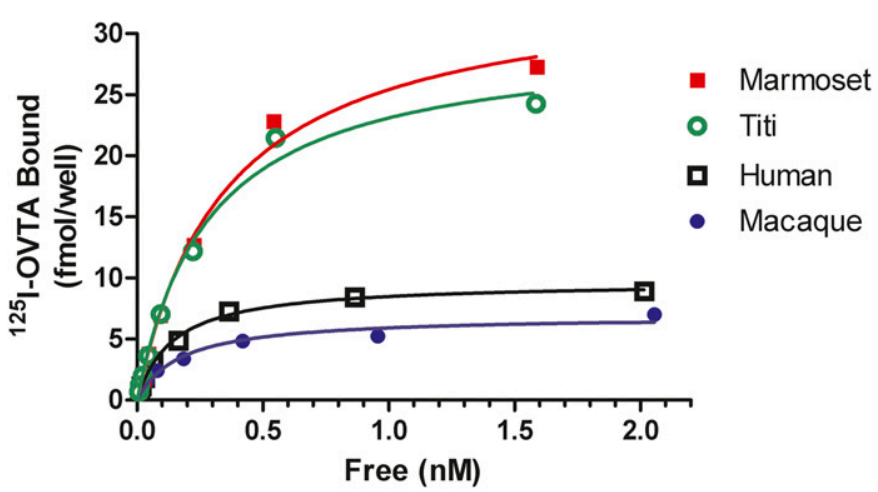

Fig. 1. Representative saturation assays for ${ }^{125}$ I-OVTA binding to OTRs from each of the four species. Cells on 96 -well plates were incubated on ice in $50 \mu$ l binding medium with the indicated concentrations of ${ }^{125}$ I-OVTA for 3 hours, and specific binding was then quantified. Data are from a single experiment with all four receptors tested side by side in triplicate. Values for this experiment are in good agreement with the average values in the Results and Table 1: humans, $B_{\max }=9.7 \mathrm{fmol} / \mathrm{well}$ and $K_{\mathrm{d}}=0.12 \mathrm{nM}$; macaques, $B_{\max }=6.9 \mathrm{fmol} / \mathrm{well}$ and $K_{\mathrm{d}}=0.144 \mathrm{nM}$; marmosets (R10), $B_{\max }$ $=34 \mathrm{fmol} /$ well and $K_{\mathrm{d}}=0.30 \mathrm{nM}$; and titi monkeys, $B_{\max }=30 \mathrm{fmol} / \mathrm{well}$ and $K_{\mathrm{d}}=0.24 \mathrm{nM}$.

plotting bound ${ }^{125}$ I-OVTA versus competitor concentration. IC $_{50}$ values were then calculated using the Cheng-Prusoff equation and each receptor's affinity for ${ }^{125}$ I-OVTA to produce $K_{\mathrm{i}}$ values. These assays were done at least three times on 3 different days using fresh aliquots of ${ }^{125} \mathrm{I}$-OVTA and $\mathrm{Leu}^{8}$-OT, Pro ${ }^{8}$-OT, and AVP for three biologic replicates per clone.

$\mathbf{C a}^{2+}$ Mobilization Assays. CHO cells expressing primate OTRs were plated into 96 -well plates and grown to $80 \%-90 \%$ confluence. On the day of assay, growth medium was aspirated and cells were incubated at $37^{\circ} \mathrm{C}$ with $100 \mu \mathrm{l}$ Fluo-4 Direct dye mixed in Fluo-4 Direct $\mathrm{Ca}^{2+}$ Assay Buffer (Thermo Fisher Scientific) with $5 \mathrm{mM}$ probenecid for 1 hour. At the end of 1 hour, baseline fluorescence was measured at $37^{\circ} \mathrm{C}$ followed by stimulated fluorescence in the presence or absence of $10^{-11}$ to $10^{-6} \mathrm{M} \mathrm{Pro}^{8}$-OT, Leu ${ }^{8}$-OT, or AVP $(3 \times$ technical replicates). Peak fluorescence minus baseline fluorescence was then plotted as a function of ligand concentration to determine $\mathrm{EC}_{50}$ values. These assays were done at least three times on 3 different days using fresh aliquots of $\mathrm{Leu}^{8}$-OT, Pro $^{8}$-OT, and AVP for three biologic replicates per clone.

Data Analysis. Binding affinities $\left(K_{\mathrm{d}}\right)$ for ${ }^{125} \mathrm{I}$-OVTA at each primate OTR were calculated by subtracting nonspecific binding and then plotting bound ${ }^{125} \mathrm{I}$-OVTA versus free ${ }^{125} \mathrm{I}$-OVTA.

Because concentrations of ${ }^{125} \mathrm{I}$-OVTA were not identical from experiment to experiment, technical replicates within each experiment $(n=3)$ were normalized and binding affinities $\left(K_{\mathrm{i}}\right)$ were calculated using the Cheng-Prusoff equation and the measured binding affinity for ${ }^{125}$ I-OVTA. Technical replicates were then averaged and used as biologic replicates ( $n=3$ per clone) to determine and compare $K_{\mathrm{i}}$ values for each ligand within species. A Bonferronicorrected cutoff $(P=0.05 \div 3=0.0167)$ was used to determine statistically significant differences in $K_{\mathrm{i}}$ values.

Within-species differences in $\mathrm{Ca}^{2+}$ mobilization potency $\left(\mathrm{EC}_{50}\right)$ were determined by normalizing and averaging each technical replicate $(n=3)$ and then using the biologic replicates $(n=3)$ to assess ligand comparisons (Pro ${ }^{8}$-OT vs. Leu ${ }^{8}-\mathrm{OT}, \mathrm{Pro}^{8}$-OT vs. AVP, and Leu ${ }^{8}$-OT vs. AVP). A Bonferroni-corrected cutoff $(P=$ $0.05 \div 3=0.0167$ ) was used to determine statistically significant differences in $K_{\mathrm{i}}$ values.

All data were analyzed using the nonlinear least-squares curvefitting capabilities of GraphPad Prism software.

\section{Results}

Saturation Binding Assays. Saturation assays were performed on 96 -well plates with $50 \mu \mathrm{l}$ binding medium per well. Representative saturation curves for all receptors are shown in Fig. 1. All of the binding and signaling assays for the human receptor were conducted with a single clone with a $B_{\max }$ value of $17 \pm 6 \mathrm{fmol} / \mathrm{well}(n=3)$. All assays for the macaque receptor were with a clone with a $B_{\max }$ value of $12 \pm$ $5 \mathrm{fmol} / \mathrm{well}(n=3)$. All assays for the titi monkey receptor were with a clone with a $B_{\max }$ value of $44 \pm 12 \mathrm{fmol} / \mathrm{well}(n=3)$. For the marmoset receptor, some assays were performed with a significantly higher expressing clone, R9, with a $B_{\max }$ value of $91 \pm 20 \mathrm{fmol} /$ well $(n=2)$; additional experiments were performed with a clone with lower expression, R10, with a $B_{\max }$ value of $33 \pm 5 \mathrm{fmol} / \mathrm{well}(n=4)$.

Saturation binding analyses revealed only relatively small differences in binding affinities for the radioligand ${ }^{125}$ I-OVTA among the four species, ranging from 161 to $481 \mathrm{pM}$ (Table 2). The human and macaque OTRs exhibited very similar affinities that were somewhat higher than those for the titi monkey and marmoset, with the marmoset exhibiting the lowest affinity.

Competition Binding Assays with OT Variants and AVP. In competition binding assays, $\mathrm{Pro}^{8}$-OT exhibited a higher binding affinity than Leu ${ }^{8}$-OT for all four species, with a 1.5-fold difference for macaques, a 2-fold difference for marmosets, a 3-fold difference for humans, and over 6-fold difference for titi monkeys. Only for titi monkeys and humans was the difference in binding affinity statistically significant $[F(1,42)>12.1, P<0.016]$. For the human OTR, the difference was due to greater affinity for $\mathrm{Pro}^{8}-\mathrm{OT}$; for the titi OTR, the difference was due to lower affinity for Leu ${ }^{8}$-OT, rather than higher affinity for $\mathrm{Pro}^{8}$-OT compared with the

TABLE 2

Binding affinities for ligands at various primate OTRs

Data are presented as means \pm S.E.M. and means $\pm \log$ S.E.M. for Kd and Ki values, respectively.

\begin{tabular}{lccccc}
\hline \multirow{2}{*}{ OTR } & \multirow{2}{*}{$n$} & ${ }^{125}$ I-OVTA $K_{\mathrm{d}}$ & \multicolumn{3}{c}{$K_{\mathrm{i}}$} \\
\cline { 4 - 6 } & & \multicolumn{1}{c}{ Pro $^{8}$-OT } & Leu $^{8}$-OT & AVP \\
\hline Human & 3 & $0.161 \pm 0.019$ & $22.78 \pm 0.10^{a}$ & $71.84 \pm 0.10$ & $541.1 \pm 0.07^{a, b}$ \\
Macaque & 3 & $0.199 \pm 0.036$ & $43.36 \pm 0.07$ & $74.99 \pm 0.08$ & $474.8 \pm 0.10^{a, b}$ \\
Marmoset & 5,6 & $0.481 \pm 0.041$ & $81.31 \pm 0.11$ & $170.2 \pm 0.10$ & $1093 \pm 0.13^{a, b}$ \\
Titi monkey & 3 & $0.289 \pm 0.031$ & $146.9 \pm 0.11^{a}$ & $894.5 \pm 0.12$ & $1924 \pm 0.16^{b}$ \\
\hline
\end{tabular}

\footnotetext{
${ }^{a}$ Indicates a significant within-species difference compared with Leu ${ }^{8}$-OT using a Bonferroni-corrected cutoff of $P<0.0167$.

${ }^{b}$ Indicates a significant within-species difference compared with Pro ${ }^{8}$-OT using a Bonferroni-corrected cutoff of $P<0.0167$.
} 

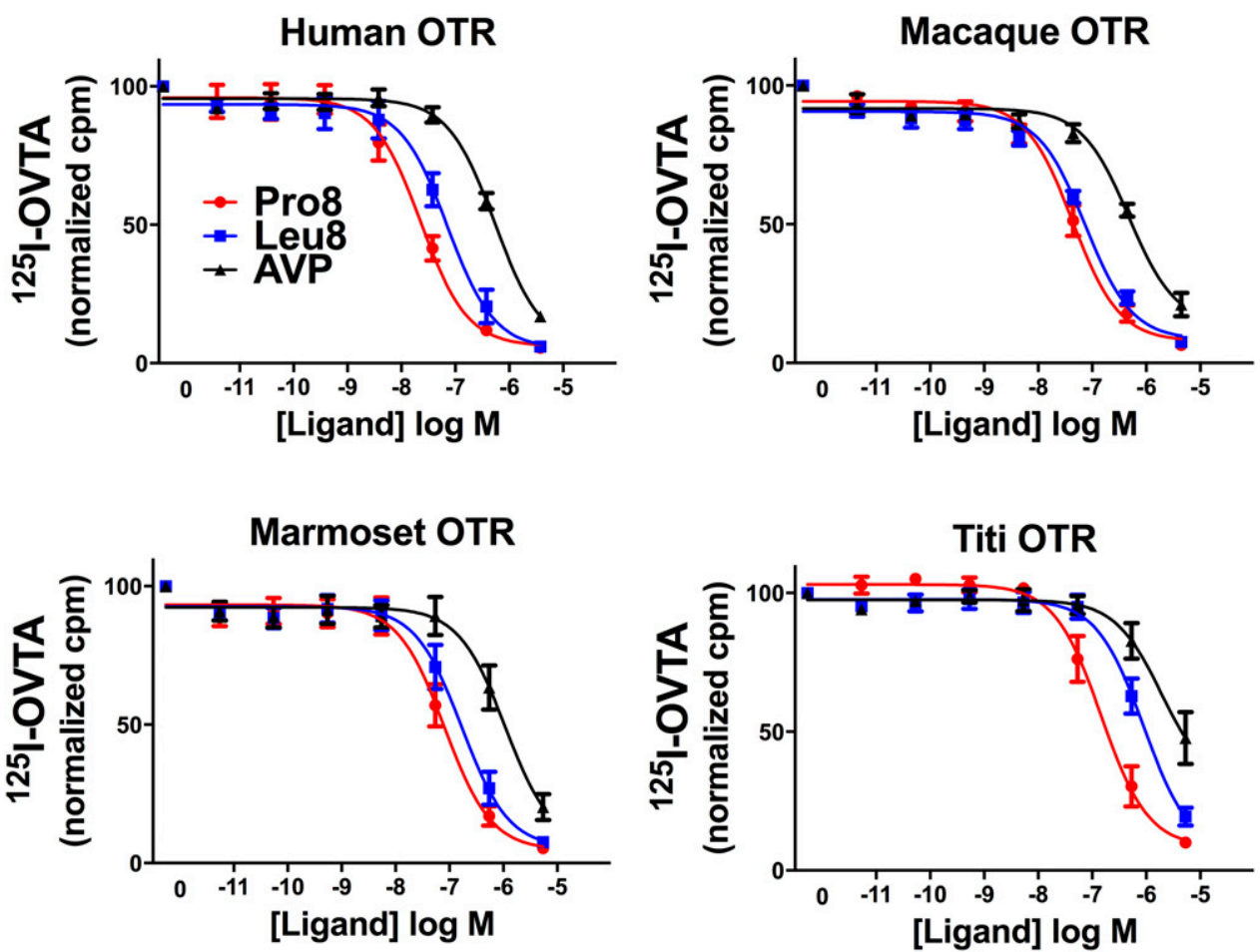

Fig. 2. Competition curves for $\mathrm{Pro}^{8}-\mathrm{OT}$ and Leu ${ }^{8}$-OT for each primate species OTR. Increasing concentrations of competitor ligand $\left(\mathrm{Pro}^{8}\right.$-OT, $\mathrm{Leu}^{8}$-OT, or AVP) were added to a constant concentration of ${ }^{125}$ I-OVTA in intact $\mathrm{CHO}$ cells expressing one of four primate OTRs. All values are expressed as the percentage of the maximal binding in the absence of OT or AVP. other species (Fig. 2; Table 2). For both OT variants, the absolute binding affinities were 3- to 5-fold higher for humans, macaques, and marmosets than for titi monkeys.

Binding affinities for AVP were assessed alongside the two OT variants for all of the receptors. Compared with $\mathrm{Pro}^{8}$-OT and Leu ${ }^{8}$-OT, respectively, binding affinity for AVP was 20- and 8-fold lower for humans, 11- and 6-fold lower for macaques, and 13- and 6-fold lower for marmosets, but 13 - and only 2 -fold lower for titi monkeys. In fact, the affinity of the titi OTR for Leu ${ }^{8}$-OT was not significantly higher than that for AVP $[F(1,42)=2.07, P=0.157]$. However, the rank order of potencies was the same for all species, with affinities for Pro8 > Leu8 > AVP.

$\mathbf{C a}^{2+}$ Signaling Assays. In $\mathrm{Ca}^{2+}$ mobilization assays, the rank order of potencies was the same for all species and with the same pattern as for binding, Pro8 $>$ Leu $8>$ AVP; however, the magnitude of the differences was smaller for signaling than for binding. Pro ${ }^{8}$ and Leu ${ }^{8}$-OT were roughly equipotent for all species, with only 1.5-fold greater potency of Pro8-OT versus Leu ${ }^{8}-\mathrm{OT}$ for humans, macaques, marmosets, and titi monkeys (Fig. 3; Table 3). Pro ${ }^{8}$-OT consistently exhibited a slightly lower maximal response than Leu8 for all species except the marmoset.

$\mathrm{Ca}^{2+}$ mobilization potencies for AVP were assessed alongside the two OT variants for all of the receptors. Compared with $\mathrm{Pro}^{8}$-OT and Leu ${ }^{8}$-OT, respectively, potency for AVP was 12- and 7-fold lower for humans, 6- and 4-fold lower for macaques, 5- and 2-fold lower for marmosets, and 8- and 5 -fold lower in titi monkeys. The absolute potencies for $\mathrm{Pro}^{8}-\mathrm{OT}$ and $\mathrm{Leu}^{8}$-OT for humans and marmosets were similar, but the potency of AVP for the marmoset receptor was nearly 2-fold higher than it was for the human receptor. Potencies for each ligand across species were higher for human and marmoset OTRs compared with the macaque and titi monkey.
$\mathrm{Ca}^{2+}$ mobilization potencies relative to binding affinities were also computed as a metric of coupling efficiency (Table 4). In general, efficiencies within species were similar, with signaling $\mathrm{EC}_{50}$ values exhibiting potencies over 2 log units higher than the binding affinities for all three ligands. The macaque OTR was the least efficient, signaling at potencies less than 2 log units higher than the binding affinity for all three ligands. Notably, in all species except the titi monkey, AVP was equally or more efficient at mobilizing $\mathrm{Ca}^{2+}$ than Pro $^{8}$-OT or Leu ${ }^{8}$-OT, per unit of binding affinity.

\section{Discussion}

The studies here tested the hypothesis that the coevolution between Pro ${ }^{8}$-OT and OTRs in NWMs (Ren et al., 2015; Vargas-Pinilla et al., 2015) would confer greater selectivity in binding and signaling for $\mathrm{Pro}^{8}$-OT over the ancestral $\mathrm{Leu}^{8}$-OT at receptors from Pro ${ }^{8}$-OT-expressing species, and conversely higher selectivity for Leu ${ }^{8}$-OT at receptors from species expressing Leu $^{8}$-OT. The binding and signaling data in this study show that this hypothesis is at best only partially supported. For the marmoset OTR, the species-native Pro ${ }^{8}-\mathrm{OT}$ bound with only modestly higher affinity and induced $\mathrm{Ca}^{2+}$ mobilization with higher potency than Leu ${ }^{8}$-OT. In humans and titi monkeys, the species-non-native ligand Pro $^{8}$-OT also bound with higher affinity than the species-native ligand $\mathrm{Leu}^{8}$-OT. For receptors from all three Leu ${ }^{8}-\mathrm{OT}-\mathrm{expressing}$ species, the two ligands were equipotent at mobilizing $\mathrm{Ca}^{2+}$. The higher binding affinity for Pro ${ }^{8}$-OT for all of the species, including those whose native hormone is Leu ${ }^{8}-\mathrm{OT}$, was unexpected and not consistent with our hypothesis of binding affinities for each species correlating with their native ligand. One explanation for the observed preference for Pro ${ }^{8}$-OT over $\mathrm{Leu}^{8}$-OT in all species may be that the flexible (Kotelchuck et al., 1972; Brewster et al., 1973) tail of Leu ${ }^{8}-O T$ can orient 


\section{Human OTR}

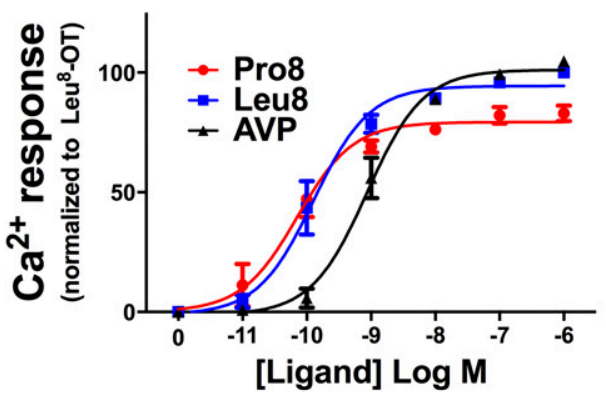

Marmoset OTR

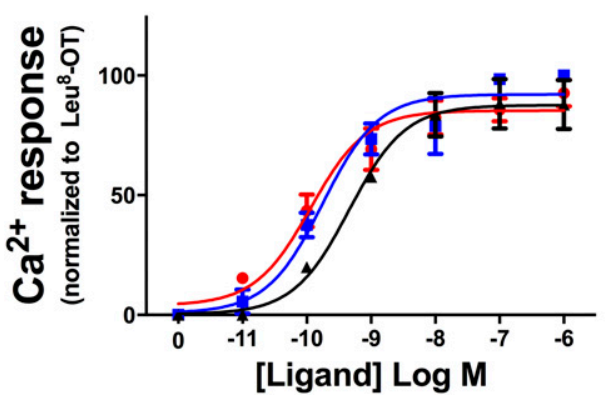

Macaque OTR

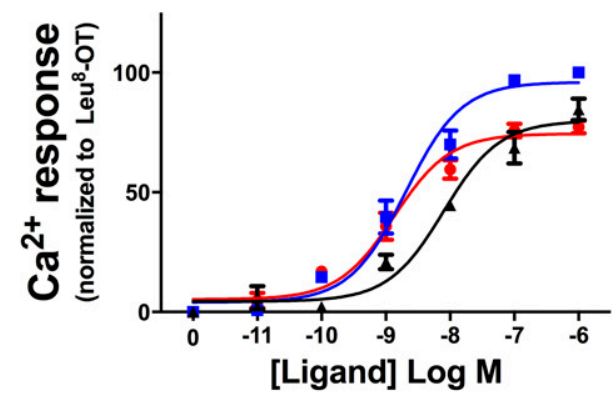

Titi OTR

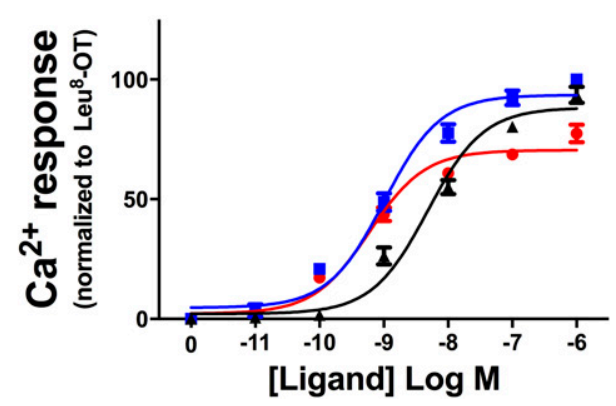

Fig. 3. Intracellular $\mathrm{Ca}^{2+}$ increases for each primate species OTR. Increasing concentrations of $\mathrm{Pro}^{8},-\mathrm{OT}$, $\mathrm{Leu}^{8}$-OT, or AVP were used to stimulate intracellular $\mathrm{Ca}^{2+}$ mobilization in $\mathrm{CHO}$ cells expressing one of four primate OTRs. All values are expressed as the percentage of the maximal response to $10^{-6} \mathrm{M} \mathrm{Leu}^{8}$-OT for each species. into a conformation that is similar to the more rigid structure of Pro ${ }^{8}$-OT (Lee et al., 2011) for only a smaller percentage of ligand-receptor interactions than $\mathrm{Pro}^{8}$-OT, and that the optimal conformation for $\mathrm{Leu}^{8}-\mathrm{OT}$ is one that is similar to the structure of Pro ${ }^{8}$-OT. The lack of significant preferences for the endogenous ligand in terms of signaling potencies was similarly unexpected. Thus, differences in other factors, perhaps downstream of the initial receptor binding and activation steps, are now the more likely explanations for the differential behavioral responses to OT in Leu ${ }^{8}-\mathrm{OT}-$ versus Pro $^{8}$-OT-expressing species.

The ability of AVP to bind and activate primate OTRs was also tested, because AVP binds and activates OTRs, and AVP is known to affect social behavior in primates, including titi monkeys and marmosets (Caldwell et al., 2008; Jarcho et al., 2011; Taylor and French, 2015; Taylor et al., 2017). In all species except the titi monkey, AVP bound with much lower affinity to the OTR than $\mathrm{Pro}^{8}-\mathrm{OT}$ or $\mathrm{Leu}^{8}-\mathrm{OT}$, and in all species AVP had lower potency for mobilizing $\mathrm{Ca}^{2+}$ than $\mathrm{Pro}^{8}-\mathrm{OT}$ or

\section{TABLE 3}

$\mathrm{Ca}^{2+}$ mobilization potencies for ligands at various primate OTRs Data are presented as means $\pm \log$ S.E.M.

\begin{tabular}{lccc}
\hline \multirow{3}{*}{ OTR $(n=3)$} & \multicolumn{3}{c}{$\mathrm{Ca}^{2+} \mathrm{EC}_{50}$} \\
\cline { 2 - 4 } & Pro $^{8}$-OT & Leu $^{8}-\mathrm{OT}$ & AVP \\
\hline & \multicolumn{3}{c}{$n M$} \\
Human & $0.072 \pm 0.12$ & $0.127 \pm 0.10$ & $0.864 \pm 0.07^{a, b}$ \\
Macaque & $1.341 \pm 0.11$ & $2.025 \pm 0.11$ & $7.981 \pm 0.12^{a, b}$ \\
Marmoset & $0.115 \pm 0.15$ & $0.176 \pm 0.14$ & $0.459 \pm 0.14^{b}$ \\
Titi monkey & $0.595 \pm 0.09$ & $1.010 \pm 0.09$ & $4.821 \pm 0.09^{a, b}$ \\
\hline
\end{tabular}

${ }^{a}$ Indicates a significant within-species difference compared with Leu ${ }^{8}$-OT using a Bonferroni-corrected cutoff of $P<0.0167$.

${ }^{b}$ Indicates a significant within-species difference compared with Pro $^{8}$-OT using a Bonferroni-corrected cutoff of $P<0.0167$.
Leu8-OT. These results show that the coevolution of Pro ${ }^{8}-\mathrm{OT}$ and the OTR in marmosets has not altered selectivity for AVP versus the two OT variants.

To our knowledge, this study is the first description of NWM OT ligand variant binding in nonhuman primates, and the $\mathrm{Ca}^{2+}$ mobilization data inform the recent research investigating OTR signaling and NWM ligand variants in rodent models. Parreiras-E-Silva et al. (2017) found no differences in $\mathrm{Ca}^{2+}$ signaling at the human OTR between Pro ${ }^{8}$-OT, $\mathrm{Leu}^{8}$-OT, or the additional NWM OT variant $\mathrm{Val}^{3} \mathrm{Pro}^{8}-\mathrm{OT}$. Our data also partially replicate those obtained by our collaborators (Pierce et al., 2016, unpublished observations), who found no difference in $\mathrm{Ca}^{2+}$ signaling between Pro ${ }^{8}-\mathrm{OT}$ and $\mathrm{Leu}^{8}$-OT at the human OTR but that Pro ${ }^{8}$-OT was more efficacious than $\mathrm{Leu}^{8}$-OT at inducing $\mathrm{Ca}^{2+}$ mobilization at the marmoset OTR. Taken together, these data indicate that the substitution of proline in place of leucine does not inhibit the $G$ protein-coupled activity in species that express the ancestral $\mathrm{Leu}^{8}$-OT. Perhaps more importantly, the $\mathrm{Pro}^{8}$ substitution confers equal or greater potency for $\mathrm{Ca}^{2+}$ mobilization in a species in which Pro ${ }^{8}$-OT is the native ligand.

These binding and signaling data provide a functional link between the genetic surveys of the OT system in NWMs and the growing body of work comparing the behavioral effects of

TABLE 4

Coupling efficiencies for ligands at various primate OTRs

\begin{tabular}{lccc}
\hline \multirow{2}{*}{ OTR } & \multicolumn{3}{c}{ Potency/Affinity Ratio $-\log \left(\mathrm{Ca}^{2+} \mathrm{IC}_{50} / K_{\mathrm{i}}\right)$} \\
\cline { 2 - 4 } & Pro $^{8}-\mathrm{OT}$ & $\mathrm{Leu}^{8}-\mathrm{OT}$ & $\mathrm{AVP}$ \\
\hline Human & 2.51 & 2.75 & 2.80 \\
Macaque & 1.51 & 1.57 & 1.77 \\
Marmoset & 2.85 & 3.00 & 3.38 \\
Titi monkey & 2.39 & 2.95 & 2.60 \\
\hline
\end{tabular}


intranasal treatment with $\mathrm{Pro}^{8}$-OT and $\mathrm{Leu}^{8}$-OT in marmosets. Pro ${ }^{8}$-OT, but not Leu ${ }^{8}$-OT, enhances a variety of pairmate-directed social approach behaviors (Cavanaugh et al., 2014, 2018). Moreover, Pro ${ }^{8}$-OT increases the amount of social behavior that an OT-treated marmoset receives from its mate and reduces sociosexual behavior directed toward individuals other than the pairmate (Cavanaugh et al., 2014; Mustoe et al., 2015). Leu ${ }^{8}$-OT does affect some social behavior in marmosets, but Leu ${ }^{8}$-OT never enhances a social behavior that $\mathrm{Pro}^{8}$-OT does not also enhance (Mustoe et al., 2018). The binding and signaling data support these behavioral findings. Pro ${ }^{8}$-OT not only bound to the marmoset OTR with greater affinity but was also modestly more potent at stimulating $\mathrm{Ca}^{2+}$ mobilization. Although it is unlikely that this difference in signaling between Pro ${ }^{8}$-OT and $\mathrm{Leu}^{8}$-OT is the only contributing factor to the behavioral differences between treatment with $\mathrm{Pro}^{8}$-OT and $\mathrm{Leu}^{8}$-OT in marmosets, it is likely at least one contributing factor.

These binding and signaling data also shed new light on the clade-wise surveys of the OXT, OXTR, and AVP V1a receptor (AVPR1A) genes in NWMs. First and foremost, our data help to explain the finding that social monogamy and the OTR coevolved in NWMs (Ren et al., 2015; Vargas-Pinilla et al., 2015), with $\mathrm{Pro}^{8}$-OT binding and $\mathrm{Ca}^{2+}$ signaling both enhanced in the socially monogamous species that expresses $\mathrm{Pro}^{8}$-OT natively. Moreover, $\mathrm{Ca}^{2+}$ signaling was not reduced by the substitution of proline for leucine at the eighth position in OTRs from Leu ${ }^{8}$-OT species. This suggests that the OTR is permissive for this substitution, providing a potential mechanism for the coevolution of $\mathrm{Pro}^{8}$-OT and OTRs in NWMs (Ren et al., 2015; Vargas-Pinilla et al., 2015). The single nucleotide substitution that produced $\mathrm{Pro}^{8}$-OT may have had modest consequences for neurotransmission, and thus the OTR may have evolved to accommodate this substitution. There is also a relationship between social monogamy and variation in the AVPR1A gene. Interestingly, in one of the only Leu ${ }^{8}$-OT NWMs that exhibits social monogamy (the titi monkey), Leu ${ }^{8}-\mathrm{OT}$ and AVP bound the OTR with similar affinity. These genetic and signaling data suggest that interrogation of the NWM AVP receptors (V1aR, V1bR, V2R) may provide new insights into nonapeptide signaling in primates, and these studies are currently in progress.

Alongside the work of Parreiras-E-Silva et al. (2017), this article constitutes a "first look" at the characteristics of the marmoset and titi monkey OTRs when bound to the Pro ${ }^{8}$-OT variant. As such, we only explored two facets of GPCR function: ligand binding and $\mathrm{Ca}^{2+}$ signaling. Other characteristics of these receptors, such as differential coupling to specific $\mathrm{G} \alpha$ subunits or bias for $\mathrm{G}$ proteins versus $\beta$-arrestin, are beyond the scope of this project but are nonetheless interesting future directions. The OTR is capable of coupling to a variety of $\mathrm{G} \alpha$ subunits resulting in a variety of cellular outcomes [see Gimpl and Fahrenholz (2001) and Mustoe et al. (2018) for detailed reviews], and there is a need for and a value to the production and characterization of ligands that are functionally selective at the OTR as tools to target specific signaling cascades. Indeed, even relatively small modifications to the OT ligand can alter the functional selectivity at the OTR, causing it to couple to different $\mathrm{G} \alpha$ subunits (Busnelli et al., 2012), and it is already known that $\mathrm{Pro}^{8}$-OT is less efficacious than $\mathrm{Leu}^{8}$-OT at promoting $\beta$-arrestin recruitment and internalization at the human receptor (Parreiras-E-Silva et al., 2017). It is possible that $\mathrm{Pro}^{8}$-OT and Leu ${ }^{8}$-OT may differentially promote coupling to specific $\mathrm{G} \alpha$ subunits or bias signaling via $\mathrm{G}$ proteins versus $\beta$-arrestin in marmosets and titi monkeys as well, and these experiments may provide more insight into the evolution of this system in NWMs. Another interesting possibility is that the OTRs may form dimers with various other GPCRs, and the $\mathrm{Leu}^{8}$-OT and Pro ${ }^{8}$-OT variants might exhibit selectivity for binding or activating one of these dimers versus another. Such dimer selectivity would not be detected in these assays with only the OTR expressed. Thus, multiple possible explanations for the ligand variation and its correlations with GPCR signaling and behavior remain to be explored.

A final potentially important outcome of these studies is that the higher binding affinity of $\mathrm{Pro}^{8}$-OT versus Leu ${ }^{8}-\mathrm{OT}$ at OTRs from all species, including humans, should presumably make $\mathrm{Pro}^{8}-\mathrm{OT}$ a better ligand for future binding studies, in either a radiolabeled or fluorescently tagged form. The 3-fold higher binding affinity would allow the use of 3-fold lower concentrations of the ligand to achieve the same fractional receptor occupancy, thus decreasing the amount of ligand required and the corresponding cost and usage of the ligand. The tighter binding of the $\mathrm{Pro}^{8}$-OT variant to the OTR may be useful in other contexts as well.

\section{Acknowledgments}

We thank Dr. Dongren Ren for constructing the titi monkey plasmid and Dr. Emily Harrison and Dr. Sara Freeman for assistance during the initial stages of this project. We also thank Dr. Thomas Murray, Dr. Marsha Pierce, and Dr. Aaryn Mustoe for input during the planning of this project.

\section{Authorship Contributions}

Participated in research design: Taylor, Schulte, French, Toews. Conducted experiments: Taylor, Schulte.

Performed data analysis: Taylor, Schulte, Toews.

Wrote or contributed to the writing of the manuscript: Taylor, Schulte, French, Toews.

\section{References}

Bakermans-Kranenburg MJ and van Ijzendoorn MH (2013) Sniffing around oxytocin: review and meta-analyses of trials in healthy and clinical groups with implications for pharmacotherapy. Transl Psychiatry 3:e258.

Beets I, Temmerman L, Janssen T, and Schoofs L (2013) Ancient neuromodulation by vasopressin/oxytocin-related peptides. Worm 2:e24246.

Brewster AIR, Hruby VJ, Glasel JA, and Tonelli AE (1973) Proposed conformations of oxytocin and selected analogs in dimethyl sulfoxide as deduced from proton magnetic resonance studies. Biochemistry 12:5294-5304.

Busnelli M, Bulgheroni E, Manning M, Kleinau G, and Chini B (2013) Selective and potent agonists and antagonists for investigating the role of mouse oxytocin receptors. J Pharmacol Exp Ther 346:318-327.

Busnelli M, Saulière A, Manning M, Bouvier M, Galés C, and Chini B (2012) Functional selective oxytocin-derived agonists discriminate between individual G protein family subtypes. J Biol Chem 287:3617-3629.

Caldwell HK, Lee H-J, Macbeth AH, and Young WS, 3rd (2008) Vasopressin: behavioral roles of an "original" neuropeptide. Prog Neurobiol 84:1-24.

Cavanaugh J, Mustoe A, and French JA (2018) Oxytocin regulates reunion affiliation with a pairmate following social separation in marmosets. Am J Primatol DOI: 10.1002/ajp.22750 [published ahead of print].

Cavanaugh J, Mustoe AC, Taylor JH, and French JA (2014) Oxytocin facilitates fidelity in well-established marmoset pairs by reducing sociosexual behavior toward opposite-sex strangers. Psychoneuroendocrinology 49:1-10.

Chan WY, Wo NC, and Manning M (1996) The role of oxytocin receptors and vasopressin V1a receptors in uterine contractions in rats: implications for tocolytic therapy with oxytocin antagonists. Am J Obstet Gynecol 175:1331-1335.

DeMayo MM, Song YJC, Hickie IB, and Guastella AJ (2017) A review of the safety, efficacy and mechanisms of delivery of nasal oxytocin in children: therapeutic potential for autism and Prader-Willi syndrome, and recommendations for future research. Paediatr Drugs 19:391-410.

Ellendorff F, Forsling ML, and Poulain DA (1982) The milk ejection reflex in the pig. J Physiol 333:577-594.

Feifel D, Shilling PD, and MacDonald K (2016) A review of oxytocin's effects on the positive, negative, and cognitive domains of schizophrenia. Biol Psychiatry $\mathbf{7 9}$ $222-233$. 
French JA, Taylor JH, Mustoe AC, and Cavanaugh J (2016) Neuropeptide diversity and the regulation of social behavior in New World primates. Front Neuroendocrinol 42:18-39.

Fuchs AR, Fuchs F, Husslein P, Soloff MS, and Fernström MJ (1982) Oxytocin receptors and human parturition: a dual role for oxytocin in the initiation of labor. Science 215:1396-1398.

Gimpl G and Fahrenholz F (2001) The oxytocin receptor system: structure, function, and regulation. Physiol Rev 81:629-683.

Gruber CW, Koehbach J, and Muttenthaler M (2012) Exploring bioactive peptides from natural sources for oxytocin and vasopressin drug discovery. Future Med Chem 4:1791-1798.

Jarcho MR, Mendoza SP, Mason WA, Yang X, and Bales KL (2011) Intranasal vasopressin affects pair bonding and peripheral gene expression in male Callicebus cupreus. Genes Brain Behav 10:375-383.

Koehbach J, Stockner T, Bergmayr C, Muttenthaler M, and Gruber CW (2013) In sights into the molecular evolution of oxytocin receptor ligand binding. Biochem Soc Trans 41:197-204.

Kotelchuck D, Scheraga HA, and Walter R (1972) Conformational energy studies of oxytocin and its cyclic moiety. Proc Natl Acad Sci USA 69:3629-3633.

Lee AG, Cool DR, Grunwald WC Jr, Neal DE, Buckmaster CL, Cheng MY, Hyde SA, Lyons DM, and Parker KJ (2011) A novel form of oxytocin in New World monkeys. Biol Lett 7:584-587.

Lee H-J, Macbeth AH, Pagani JH, and Young WS, 3rd (2009) Oxytocin: the great facilitator of life. Prog Neurobiol 88:127-151.

Lockard MA, Ebert MS, and Bargmann CI (2017) Oxytocin mediated behavior in invertebrates: an evolutionary perspective. Dev Neurobiol 77:128-142.

Manning M, Misicka A, Olma A, Bankowski K, Stoev S, Chini B, Durroux T, Mouillac B, Corbani M, and Guillon G (2012) Oxytocin and vasopressin agonists and antagonists as research tools and potential therapeutics. $J$ Neuroendocrinol 24:609-628.

McNeilly AS, Robinson ICAF, Houston MJ, and Howie PW (1983) Release of oxytocin and prolactin in response to suckling. Br Med J (Clin Res Ed) 286:257-259.

Mustoe A, Taylor JH, and French JA (2018) Oxytocin structure and function in New World monkeys: from pharmacology to behavior. Integr Zool DOI: 10.1111/1749 4877.12318 [published ahead of print].

Mustoe AC, Cavanaugh J, Harnisch AM, Thompson BE, and French JA (2015) Do marmosets care to share? Oxytocin treatment reduces prosocial behavior toward strangers. Horm Behav 71:83-90.

Muttenthaler M, Andersson A, Vetter I, Menon R, Busnelli M, Ragnarsson L, Bergmayr C, Arrowsmith S, Deuis JR, Chiu HS, et al. (2017) Subtle modifications to oxytocin produce ligands that retain potency and improved selectivity across species. Sci Signal 10:eaan3398.

Parker KJ, Oztan O, Libove RA, Sumiyoshi RD, Jackson LP, Karhson DS, Summers JE, Hinman KE, Motonaga KS, Phillips JM, et al. (2017) Intranasal oxytocin treatment for social deficits and biomarkers of response in children with autism. Proc Natl Acad Sci USA 114:8119-8124.

Parreiras-E-Silva LT, Vargas-Pinilla P, Duarte DA, Longo D, Espinoza Pardo GV Dulor Finkler A, Paixão-Côrtes VR, Paré P, Rovaris DL, Oliveira EB, et al. (2017) Functional New World monkey oxytocin forms elicit an altered signaling profile and promotes parental care in rats. Proc Natl Acad Sci USA 114:9044-9049.

Postina R, Kojro E, and Fahrenholz F (1996) Separate agonist and peptide antagonist binding sites of the oxytocin receptor defined by their transfer into the V2 vasopressin receptor. J Biol Chem 271:31593-31601.

Ren D, Lu G, Moriyama H, Mustoe AC, Harrison EB, and French JA (2015) Genetic diversity in oxytocin ligands and receptors in New World monkeys. PLoS One 10: e0125775.

Sawyer WH and Manning M (1973) Synthetic analogs of oxytocin and the vasopressins. Annu Rev Pharmacol 13:1-17.

Taylor JH and French JA (2015) Oxytocin and vasopressin enhance responsiveness to infant stimuli in adult marmosets. Horm Behav 75:154-159.

Taylor JH, Intorre AA, and French JA (2017) Vasopressin and oxytocin reduce food sharing behavior in male, but not female marmosets in family groups. Front Endocrinol (Lausanne) 8:181.

Vargas-Pinilla P, Paixão-Côrtes VR, Paré P, Tovo-Rodrigues L, Vieira CM de AG, Xavier A, Comas D, Pissinatti A, Sinigaglia M, Rigo MM, et al. (2015) Evolutionary pattern in the OXT-OXTR system in primates: coevolution and positive selection footprints. Proc Natl Acad Sci USA 112:88-93.

Wallis M (2012) Molecular evolution of the neurohypophysial hormone precursors in mammals: comparative genomics reveals novel mammalian oxytocin and vasopressin analogues. Gen Comp Endocrinol 179:313-318.

Address correspondence to: Myron L. Toews, Department of Pharmacology and Experimental Neuroscience, University of Nebraska Medical Center, Durham Research Center, Room 3042, 985800 Nebraska Medical Center, Omaha, NE 68198-5800. E-mail: mtoews@unmc.edu; or Jack H. Taylor, Department of Psychology, Callitrichid Research Center, University of Nebraska at Omaha, Omaha, NE 68182. E-mail: jhtaylor@unomaha.edu 